\title{
Concept for Mobility and Interconnections Aspects in Converged NGN-Based IPTV Architecture
}

\author{
Eugen Mikoczy, Radovan Kadlic, and Pavol Podhradsky \\ NGNlab, Department of Telecommunications, FEI, Slovak University of Technology, Ilkovičova 3, 81219 Bratislava, Slovakia
}

Correspondence should be addressed to Eugen Mikoczy, mikoczy@ktl.elf.stuba.sk

Received 9 February 2009; Accepted 2 November 2009

Recommended by Alessandro Vanelli-Coralli

\begin{abstract}
The progress and evolution trends in the area of ICT and ICT infrastructure based on convergence processes create new opportunities. Service providers and network operators can provide a wide spectrum of multimedia services and applications to end users. The IPTV services represent a specific group of multimedia services which are in the sphere of interest of the telecommunication technical community but also subscribers. Standardization bodies like ETSI TISPAN or ITU-T have defined standards for NGN-based IPTV architecture and services (IMS and non-IMS). The paper evaluates possibilities and potential architecture for concept of converged NGN IPTV. New vision of the converged NGN IPTV architecture is presented together with proposed enhancements compared to IMS-based IPTV where single converged platform can serve fixed, mobile, or wireless terminals. The concept for IPTV service roaming with mobility support and different interconnection scenarios are discussed with intention to show potential user benefits.
\end{abstract}

Copyright (C 2009 Eugen Mikoczy et al. This is an open access article distributed under the Creative Commons Attribution License, which permits unrestricted use, distribution, and reproduction in any medium, provided the original work is properly cited.

\section{Introduction}

The TV service started in last century as analogue terrestrial broadcasting service. Digital television as the terrestrial or satellite service started several years ago. The IPTV could be considered as second generation of the digital television.

The standardization institutions like the ITU-T (International Telecommunication Union) and ETSI (European Telecommunications Standards Institute) TISPAN (The Telecoms \& Internet converged Services \& Protocols for Advanced Networks) began with standardization process focused on convergence processes of the circuits switched and IP networks several years ago. First version of NGN concept was proposed as more voice oriented. The NGN architectures have been designed to provide a wide spectrum of NGN multimedia services and now these technologies also support Internet Protocol Television (IPTV) services. The IPTV is defined [1] as the set of multimedia services such as television/video/audio/text/graphics/data delivered over IPbased networks. NGN IPTV network supports the required level of QoS/QoE, security, interactivity, and reliability.

In early 2008, the ETSI TISPAN NGN in Release 2 defined two main architectural concepts: NGN-dedicated
IPTV subsystem (NGN but not IMS solution) and IMSbased IPTV. ITU-T has identified also additional possible NGN-based IPTV architecture called the Converged NGNbased IPTV. Our vision of Converged NGN-based IPTV architecture is presented in the paper. The concept could be considered as evolution of IMS-based IPTV where single platform can be used to deliver IPTV over multiple type of access network (fixed-mobile converged IPTV). Selected scenarios supporting the mobility, interconnection, and service roaming within NGN-based IPTV networks are also presented.

\section{NGN-Based IPTV Architecture}

In the ETSI TISPAN NGN Release 2 and 3, several specifications of stage 1 (requirements) and stage 2 (architecture) the IPTV integration within TISPAN NGN standards is addressing are

(i) IPTV service requirements [2],

(ii) NGN integrated IPTV subsystem architecture [3] (formally NGN-dedicated IPTV in R2),

(iii) IMS support for IPTV architecture [4]. 


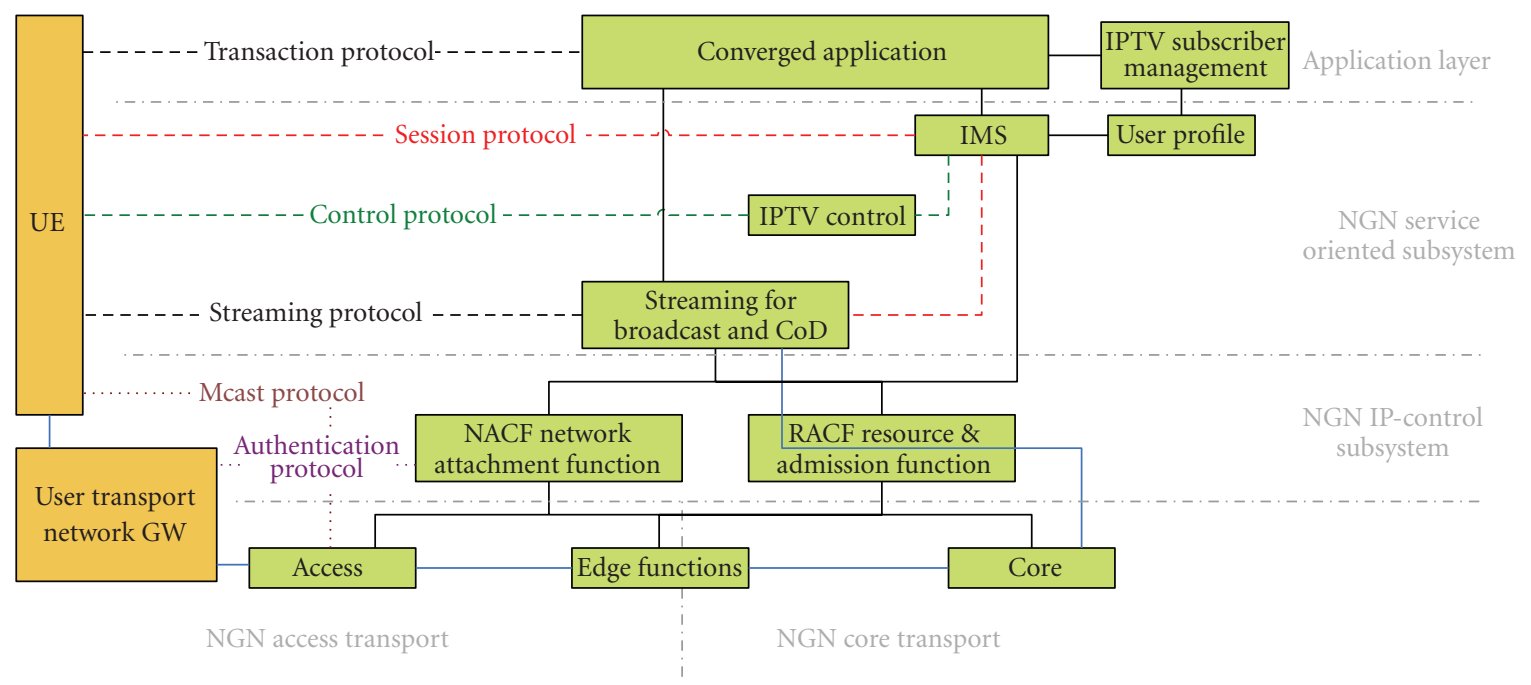

Figure 1: ITU-T concept of Converged NGN-based IPTV architecture [1].

The specifications about the implementation of the IPTV functions, interfaces, procedures, protocol recommendations (stage 3) have been finalized and more information for NGN-dedicated IPTV can be found in ETSI TS 183064 [5] or for IMS-based IPTV in ETSI TS 183063 [6]. The ITU-T NGN-based IPTV architecture is specified in recommendation of ITU-T Y.1910 named IPTV functional architecture [1]. Also other institutions like ATIS or Open IPTV forum are working on NGN-based IPTV.

IMS-based IPTV is new concept where first implementations based on ETSI TISPAN R2 have been already tested by MSF in 2008. ETSI is hosted in 2009 also IPTV plugtest where vendors and operators can test real interoperability of IMS-based IPTV servers and platforms (based on TISPAN R2 [6]). The most comprehensive set of new IPTV services will be implemented based on TISPAN Release 3 NGN-based IPTV [4].

Several scenarios are possible and really depend only on the operator's choice in which solution and migration scenarios are selected (if any) [7, 8]. The above mentioned ITU-T IPTV has been proposing the converged application framework where the non-IMS IPTV merges with the IMSbased IPTV architecture. But in this case just as purely unity of all elements supporting all interfaces from both architectures that make no sense from the complexity perspective, but also for both architectures if used in parallel, they are able to provide similar services (Figure 1).

ETSI TISPAN in release 3 (in [3] in informative annex) proposed possible migration scenarios between non-NGN, NGN non-IMS and NGN IMS-based architecture. There is also mentioned the last evolution step called converged NGN-based IPTV (this concept is not described in Release 3 because for now it is out of the scope). The following sections will describe potential concept of such architecture with additional goal to describe also the way of adaptation with multiple types of content sources, but also with multiple access and distribution networks converged to a single functional architecture.

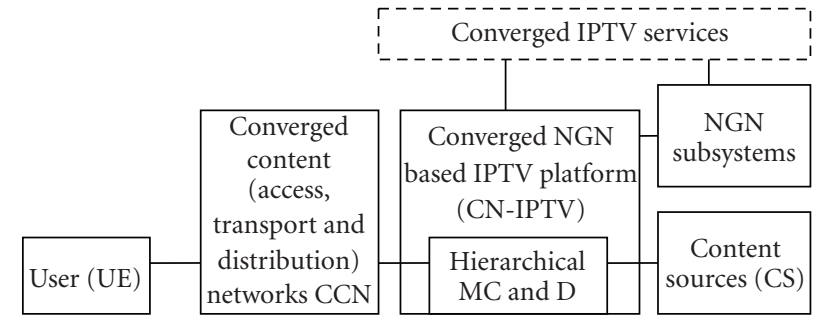

FIgure 2: High-level view to IPTV domains.

\section{Concept of Converged NGN-Based IPTV}

The concept of converged NGN-based IPTV can be split into several subsystems and domains where each one plays an important role to provide the converged approach of multiservice NGN architecture (Figure 2):

(i) content sources,

(ii) converged NGN-based IPTV architecture-overall concept, description of functionalities,

(iii) concept of hierarchical content control and delivery subsystem,

(iv) content transport and distribution networks,

(v) converged IPTV services.

3.1. Functional Architecture for Converged NGN-Based IPTV. The proposed Converged NGN-based IPTV architecture (CN-IPTV) is an evolution of the IMS-based IPTV from ETSI TISPAN Release 3. We combined IPTV service control function (ISCF) for service control which can use the IMS for specific cases but not all signaling traffics need to pass core IMS (which improve performance and shorter delays). The concept is including media control and delivery organized in hierarchical MC\&D architecture that is extended with specialized types of MDFs [1]. Additionally we elaborate possible integration with some DVB [9], 3GPP UMTS, 
and OMA BCAST component that can extend the mobility capabilities for IPTV services. DVB specified how DVB services could be provided over IP networks in specification called DVB-IPTV (formally DVB-IPI) and discussed also possible compatibility with TISPAN NGN-based IPTV [10]. Open Mobile Alliance defined service enabler primarily for mobile-related broadcasted/multicasted services (OMA BCAST [11]) but it could be used also as a base for adaptation of several types of access technologies to our proposed concept for converged NGN-based IPTV. OMA BCAST 1.1 explains the adaptation of the DVB-H, 3GPP MBMS, and WiMAX access technologies.

The proposed converged NGN-based IPTV architecture consists from following elements (Figure 3):

(i) ICAF: IPTV-Converged Application Functions,

(ii) SDSF: Service Discovery and Selection Functions,

(iii) ISCF: IPTV Service Control Function,

(iv) UPSF: User Profile Server Function (existing one),

(v) IMCF: IPTV Media Control Function,

(vi) IMDF: IPTV Media Delivery Function (P-Proxy, S-Serving, I-Interconnection),

(vii) IMS: IP Multimedia subsystem (P-/S-/ICSCF),

(viii) NASS: Network Attachment Subsystem,

(vx) RACS: Resource and Admission Control Subsystem.

Any IPTV architecture is not fully completed without other functionalities which we have also included in the proposed concept:

(i) IPTV supporting function (e.g., content preparation and manipulation),

(ii) IPTV management functions (e.g., content management),

(iii) IPTV security functions (e.g., content protection, IPTV service protection),

(iv) IPTV charging (based on NGN charging for online/offline charging but enhanced for IPTV specific scenarios),

(v) Interworking or interfacing with other NGN subsystems.

Three types of MDFs are introduced by hierarchical MC\&D. MDFs are split into multiple specific functionalities with three architecture components described [8] as follows.

Interconnection-IPTV Media Delivery Function (IIMDF): this element handles the media import and ingress of content from multiple content sources (ingress of media, metadata, content provider information, and interconnection to external domains):

(i) IPTV Headend or from content providers/originators or broadcasters, (ii) from other IPTV service providers in case of interconnection or roaming or as offer of the content from service provider playing a role of content aggregator or Media Content Distribution Network (MCDN),

(iii) from the Internet sources like the Web-based TV or from the end users like user generated content,

(iv) the IMDF need to hide the IPTV service provider infrastructure for external domains but also provide necessary functionality to interconnect to heterogeneous content sources (which can hold a variety of coding, transport, signaling schemas) and convert to content/metadata/signaling to formats supported by Converged NGN-based IPTV.

Serving-IPTV Media Delivery Function (S-IMDF): this element handles the processing of contents (e.g., encoding, content protection and transcoding) and it is also responsible for the storage of contents and metadata as well as the propagation of content information. S-IMDF provides centralized oriented services such as Content on demand for long tail content (less popular content) or recording/storing of user independent content (n-PVR or Time shifted TV or Near CoD).

Primary-IPTV Media Delivery Function (P-IMDF): this element is the primary contact point of the users which provides also the streaming and downloading functionalities for all IPTV services according to the required quality, format, and the type of casting (multi/uni/broad-casting) for particular user's end device and network accessing to P-IMDF (personalization of user specific content delivery). This element could also store the most frequently accessed CoD assets or user's specific contents (specific user n-PVRs, user generated content). The P-IMDF could be responsible for the adaptation of IPTV service delivery to other access technologies or distribution networks.

(i) Preferred way is that the P-IMDFs are located as near as possible to UE, for example, near to edge of the network. These elements can be combined with specific elements of access network (e.g., in case of using OMA BCAST and 3GPP MBMS also with integrated control elements, in case of MBMS, e.g.). P-IMDFs can also integrate Broadcast-Multicast Service Centre (BM-SC). The BM-SC provides functions needed for user service provisioning and content delivery in MBMS capable UMTS. P-IMDF integration with 3GPP MBMS and PSS helps to provide IPTV services delivery over exiting UMTS mobile network.

(ii) P-IMDF can also support mobility and seamless handover between different technologies.

(iii) P-IMDF may require to transcode or adapt the content to required bitrate, codec, or content encapsulation to specific transporting technologies.

(iv) It can be used as security elements for the content protection (e.g., digital rights management and content encoding).

The element responsible for the service discovery and selection functions (SDSFs) could support multiple formats 


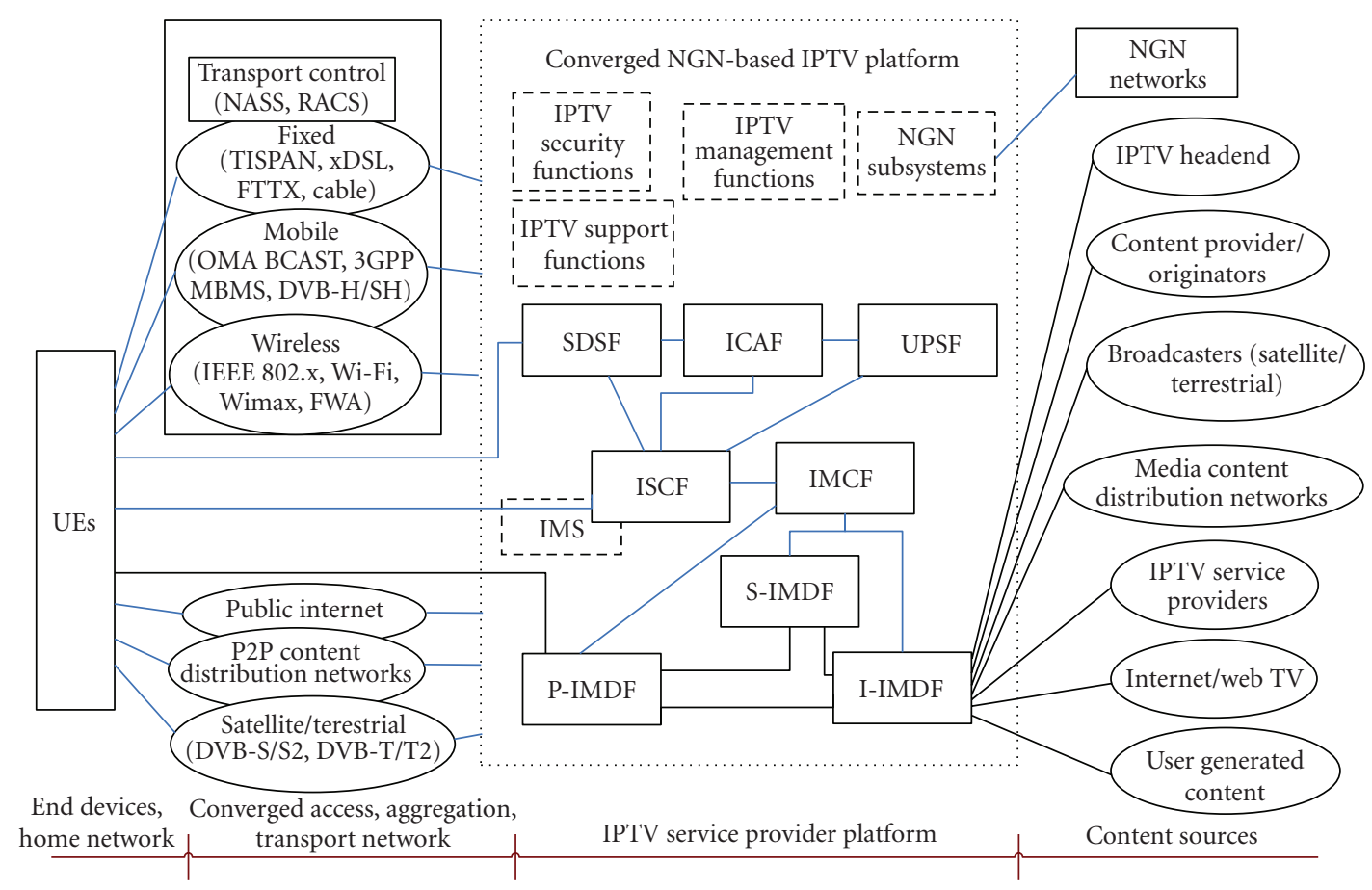

FIGURE 3: Proposed conceptual architecture for Converged NGN-based IPTV.

and mechanisms. But the main enhancement in proposed architecture is the SDSF's potential to aggregate metadata information from multiple sources (e.g., content provider, electronic program guide provider, internet, broadcasted service information) and provide them everywhere to the UE in personalized manner and allows them to integrate with other relevant information (presence, statistics, recommendations, etc.), too.

The last but not least element is (for sure) the IPTV converged application function (ICAF) which could provide combinational services and converged services with enhanced service logic and service orchestration. The ICAF can interact with other NGN application servers and subsystems and can be used for personalized service behavior based on user's preferences and settings.

The enhanced service control entity called IPTV Service Control Function (ISCF) is responsible (in conjunction with core IMS) for service control and for providing basic IPTV services and service interaction and also supports mobility on service control and application layers. Similar to other NGN-based IPTVs also the converged one needs to ensure relevant resource allocation and QoS handling (using existing interfaces to transport control). Main goal of Converged NGN-based IPTV is to provide the flexible service provider platform for IPTV services (and any NGN services as well) that may deliver personalized services over multiple access network with nomadic or seamless mobility. But we have to differentiate the IPTV service provider access infrastructure with guarantied quality (fixed technology as xDSL or FTTx, mobile technology like UMTS with IMSbased MBMS/PSS or wireless access technologies like WiFi or Wimax) from other additional distribution possibilities, like public internet (without QoS and unpredictable conditions), terrestrial/satellite distribution (mainly unidirectional for broadcasting with other technologies used for interaction, back channel signaling or unicast services), or the P2P content distribution network.

3.2. Protocol Stack for Converged NGN-Based IPTV. The protocol stack for such a complex system as Converged NGN-based IPTV platform will include almost all existing NGN protocols (Figure 4). Common layer for all of them is Internet Protocol (IP) which should be carried over different physical and data link technologies. Additionally, to IP-based NGN protocol family we can transmit the media over other type of broadcasting networks like DVB-T or DVB-S (where adaptation and other ways for interactive services and back channels for signaling may be needed).

\section{Interconnection Models and Roaming Scenarios}

In general, several scenarios with different issues that have to be solved for IMS-based IPTV or its evolution in form of Converged NGN-based IPTV for service roaming are possible. The network of the provider where the subscriber has made a contract for some service will be called the Home Network. The network of the provider where subscriber is just connected during roaming will be called the Visited Network. Main goal to support interconnection and roaming scenarios for IPTV is that given operator can interconnect other operators network over standardize interfaces to provide roaming or aggregate content and also IPTV services 


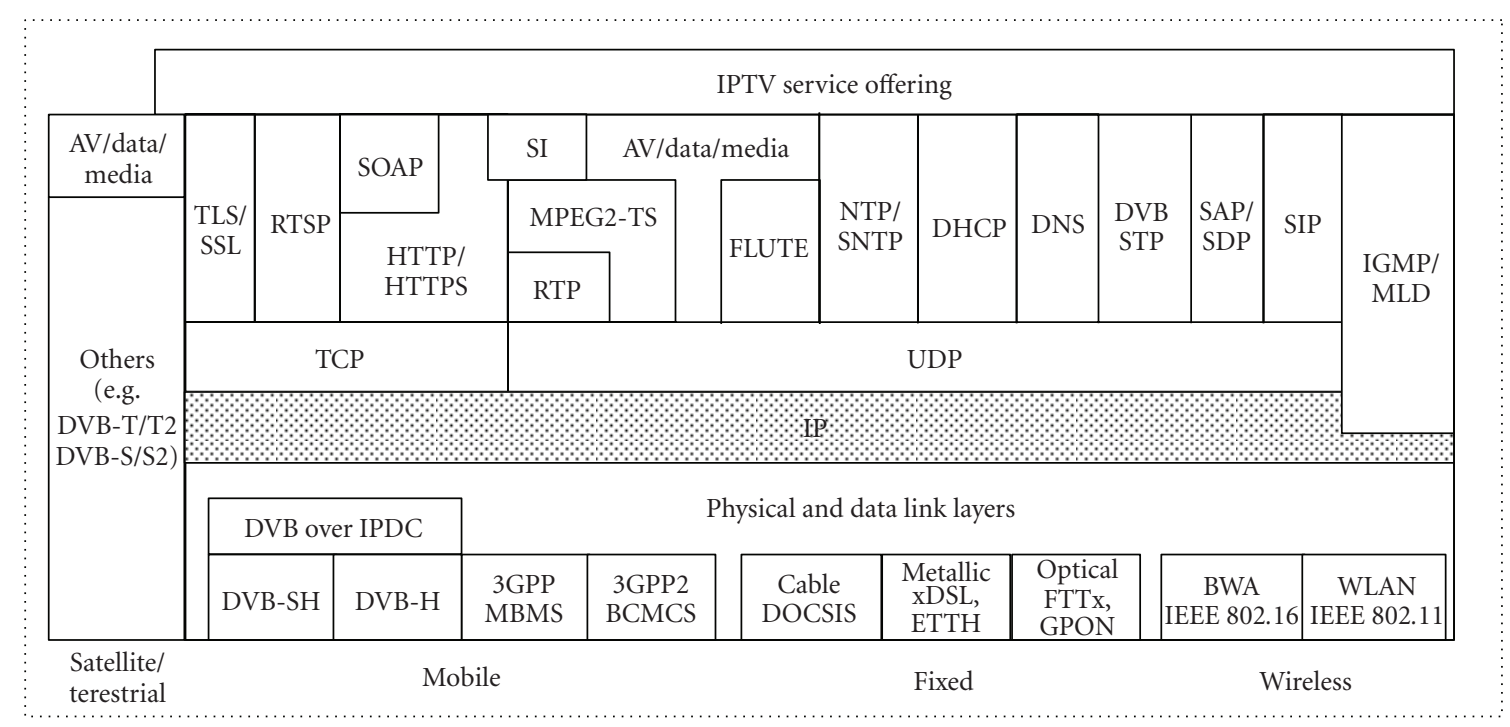

FIGURE 4: Protocol stack for Converged NGN-based IPTV platform.

which he could not provide by own platform (acquire them from partner operator). From user perspective main advantage is accessing his services outside of the Home Network or on move. The IPTV roaming also allows more variety of accessible local content (present only in Visited Network).

We can recognize at least the following basic scenarios for roaming and interconnection to home network (defined in [4] Annex H: Interconnection Models to support of Mobility Capabilities):

(i) remote data access to IPTV/content provider,

(ii) IMS interconnect to home IPTV provider,

(iii) visited-home network roaming between IPTV providers (served only from home network),

(iv) visited-home network roaming between IPTV providers (served from home or visited network).

There are several aspects which are crucial to solve the issues related to interconnection (from technical but also from service level agreement point of view):

(i) services which could be provided in roaming, if possible by partial coverage within Visited Network,

(ii) support for unicast, multicast, bandwidth negotiation, QoS issues,

(iii) model for storage, streaming, proxy of content between both domains,

(iv) service discovery and selection issues appearing from cross domain transport,

(v) content management (and generally IPTV management aspects),

(vi) IPTV security aspects,

(vii) roaming behavior, (viii) content adaptation to support visited network conditions,

(ix) multi-multicast versus transcoding for multiple access technology,

(x) codec/transport/interconnection agreed between providers,

(xi) multi-multicast versus transcoding for multiple access technology,

(xii) codec/transport/interconnection agreed between providers.

\subsection{Scenarios for Interconnect in NGN-Based IPTV}

Scenario A: IMS-IPTV Subscriber Visiting Packet/Switched Provider without IMS. Because the Visited Network has no functions of the IMS, the subscriber must use some remote data IP connection (e.g., VPN or secure remote access) to connect to his Home Network. Over this connection there can be transferred all media and signaling to the subscriber directly from his Home Network. Because such a connection should go over any IP network (also best effort) also without resource reservation mechanisms, no QoS can be really ensured. This scenario may be used for IPTV services without guaranty like remote control or download of n-PVR recordings.

Scenario B: IMS-IPTV Subscriber Visiting IMS Provider without IMS-IPTV Solution. This scenario is the simplest one with IMS involved. Subscriber will use all IPTV elements from his Home Network. The elements of visited network must provide just few functions.

(i) Check if certain subscriber is allowed to roam (via IMS roaming) to his visited network (Network-toNetwork check). 
(ii) Collect and watch all data necessary for charging.

(iii) Control limitation of its own network and apply limits to subscriber based on roaming policy agreed by Hosted and Visited Network operators.

The quality of the IPTV service for the end customer is the same one as in home network, but no reuse of local media resources for the provider is possible (because in visited network there is no NGN-based IPTV platform).

Scenario C: IMS-IPTV Subscriber Visiting IMS Provider with IMS-IPTV Solution (Only Home Served). Additionally, to the previous scenario the following one expects the IMS-based IPTV platform in Visited Network; however all content and services are delivered only from Home Network. From the Visited Network, there could be used only some supporting elements like SDSF or IMDF. Useful functions are that ones like transcoding and content adaptation to adapt to media with parameters required in Visited Network.

Scenario D: IMS-IPTV Subscriber Visiting IMS Provider with IMS-IPTV Solution (Home or Partially Visited IPTV Platform Served). If there is an IPTV solution in Visited Network, it is possible that it has similar content of CoD, Bcast, PVR service. It is useful to use this content from local resource for the visiting subscriber. The content which is available in Visited Network is not needed to be transferred over an interconnection network. Therefore the interconnect bandwidth can be saved. For this purpose both providers must agree on the same identification of content, sharing service discovery and selection information, content security, billing clearing, and for roaming agreements (e.g., QoS, Service Level Agreement). It is possible for the provider to reuse the local media resources from Visited Network.

In the following section we will focus only on the most important scenarios B, C, and D which involve the IMS and these scenarios are therefore also applicable for Converged NGN-based IPTV.

4.1.1. Scenario B: IMS-IPTV Subscriber Visiting IMS Provider without IMS-IPTV Solution. The model of interconnection with the Visited Network within the Core IMS involved is used only for IMS type of roaming to access home IPTV service provider platform [4] (Figure 5).

The UE can request the IPTV services from the Home Network when connected the Visited Network. The Core IMS in the Visited Network or Home Network can request resources from the RACS in the Visited Network through the interface Gq. The UE can be attached to the Visited Network through the interface el so that the NASS in the Visited Network can assign the IP address for the UE and discover the address of P-CSCF in the Visited Network.

The Core IMS in the Visited Network can transfer the IPTV service request from the UE through the interface $\mathrm{Gm}$ to the home Core IMS through the Ic connection of IBCFs (Intermediate Breakout Control Functions). The UE can connect to home SDSF through the interface Xa. To get configure parameters in ISCF through the interface Ut.
Service initiation, control as well as media delivery, the UE can connect over existing interfaces the Home Network.

4.1.2. Scenarios C/D: IMS-IPTV Subscriber Visiting IMS Provider with IMS-IPTV Solution. The model of interconnection with the Visited Network based on the Core IMS and Converged NGN-based IPTV infrastructure (or IMSbased IPTV) is used for most advanced type of roaming to access the home IPTV service provider platform (also with CN-IPTV) where all services are provided only from Home Network (Scenario C) or from elements from both Home/Visited networks as shown on Figure 6 (with using IMDFs in Visited Network and I-IMDFs for interconnect and content adaptation on edge of both domain).

The UE can attach to the Visited Network through the interface e1/e3 towards the NASS in the Visited Network that can assign the IP address for the UE and discover the address of P-CSCF in the Visited Network. The Core IMS in the Visited Network or Home Network can request resources from the RACS in the Visited Network through the interface Gq. The Core IMS in the Visited Network transfers the entire IPTV service request from the UE through the interface Ic to the Core IMS in the Home Network through the connection of their own IBCF (Intermediate Breakout Control Function).

The UE is connected to the Home SDSF (which can provide information about available content in this particular roaming case, because some of content could be restricted for roaming) to acquire service selection information through the interface Xa. The UE can be connected to the SCF in the Home IPTV network to configure parameters through the interface Ut (not shown on figure). The Core IMSs in both networks connect their own UPSF to get the user. But Home UPSF is responsible for service initiation authorization and user profile information required for personalization of IPTV services. The service initiation and control is provided over existing interfaces from Home Network (Gm to IMS and then other to interfaces towards ISCF, ICAF, or IMCF). The setup of media control and delivery channels is responsibile for the home ISCF with IMCF but media delivery itself could be provided from IMDFs from Home or Visited Network (we elaborate specific cases in Section 4.2) via Xc (for control) or Xd (for delivery). The End User has to subscribe the roaming services at the Home Network before he/she moves to the Visited Network. The exact details and mechanism agreed between IPTV service providers have to be negotiated in advance together with interconnect agreements, policy rules, and most probably also service level agreements (SLAs) to assure QoS on interconnect.

Following steps are proposed as potential procedures for NGN-IPTV interconnection with the Visited Network with CN-IPTV is presented (Figure 7).

(1) Network Attachment: in this step the UE attaches to the network (with NASS, receiving IP configuration, P-CSCF address discovery of Visited Network, etc.).

(2) UE performs IMS Registration in Visited Network. P-CSCF within Core IMS in Visited Network submits the registration request to the I-CSCF within the Core IMS in the Home Network. 


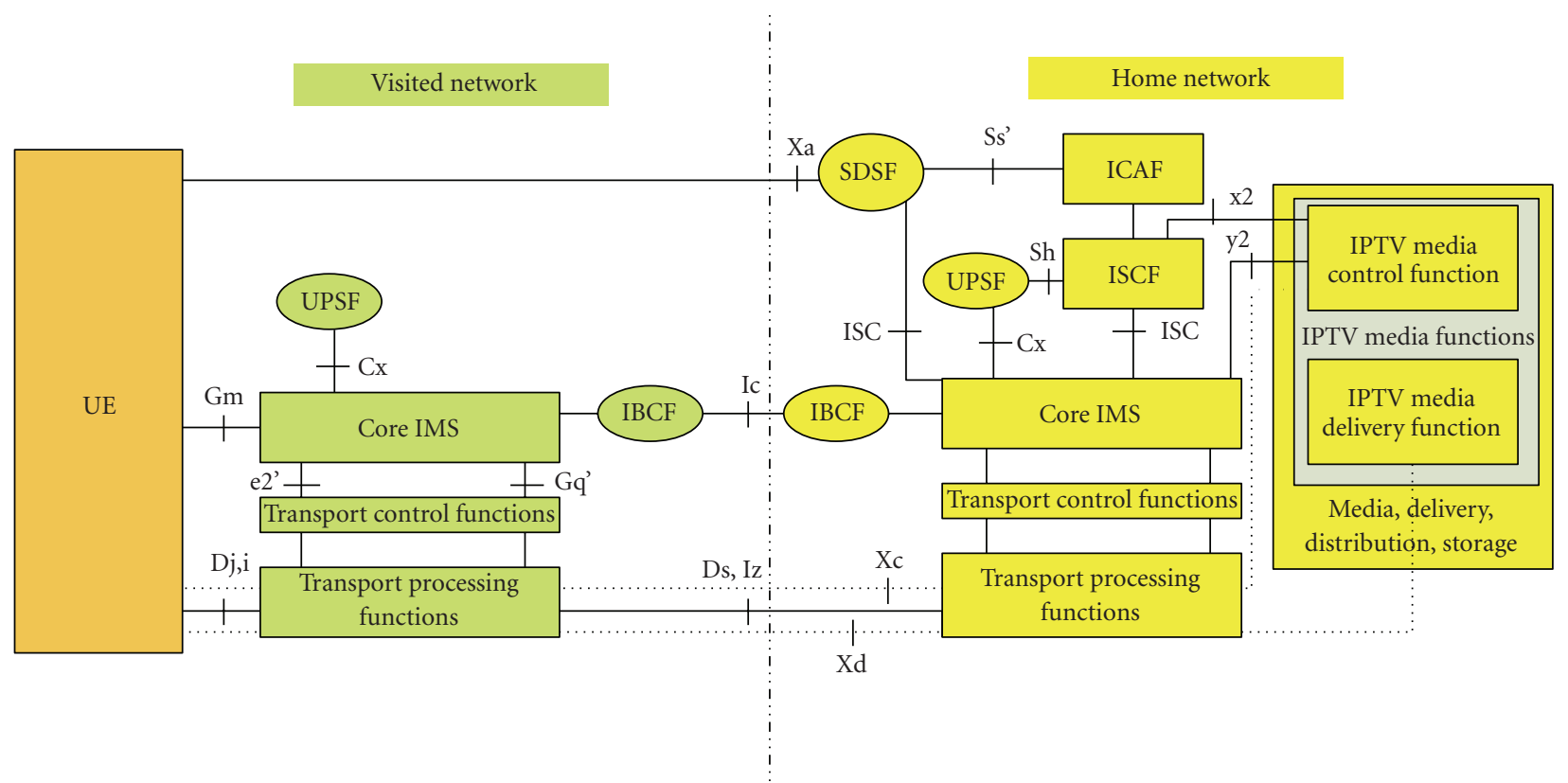

FIgURE 5: The model of interconnection with the Visited Network within Core IMS involved.

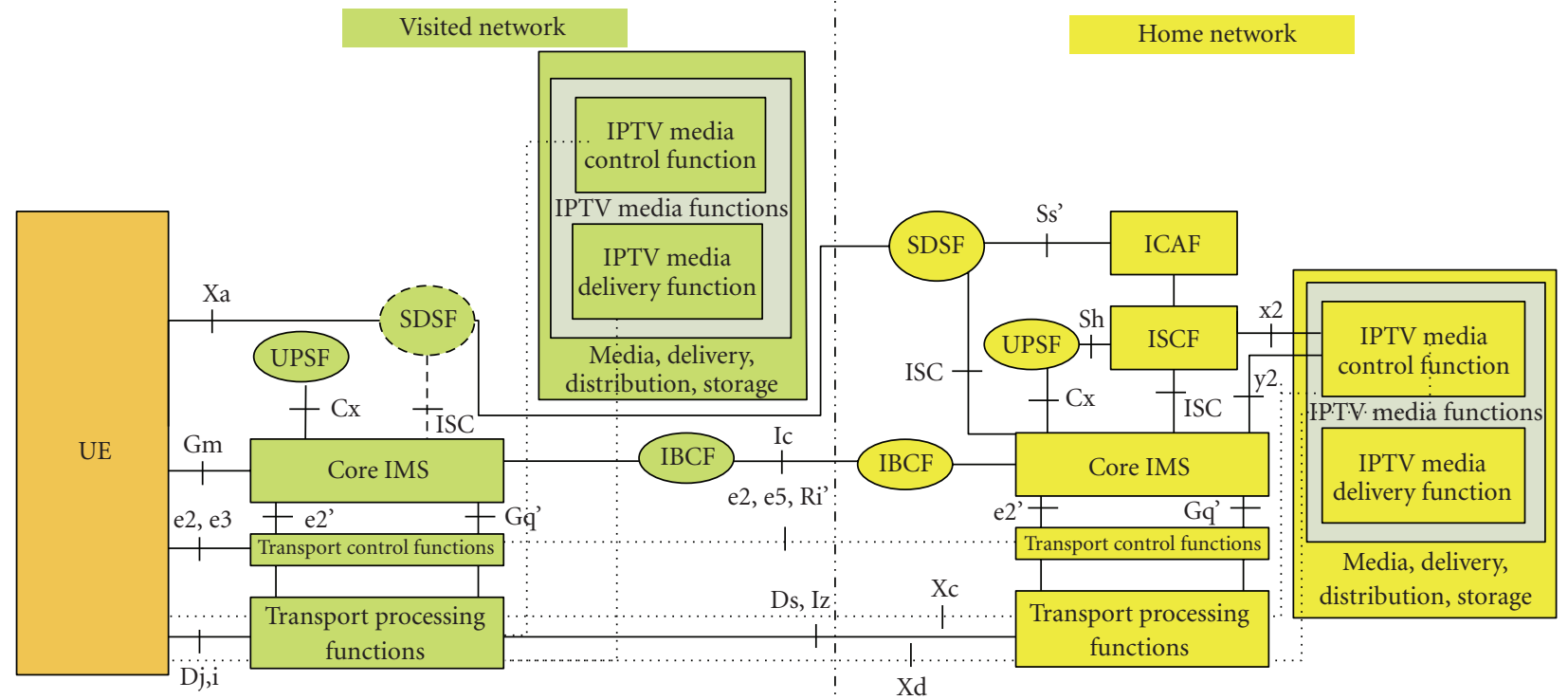

Figure 6: Model of interconnection with the Visited Network with CN-IPTV.

(3) Core IMS in the Visited Network returns parameters (e.g., P-CSCF address of Home Network, SDSF) to the UE,

(4) Home and visited network elements can require some exchanges of relevant information before allowing user and his/her terminal attached to IPTV services and select or initiate any service,

(5-6) UE performs service discovery and service selection contacting home SDSF directly or via visited SDSF (to compile a set of services and metadata which can be provided to users).

(7) User initiated via UE requests for selected service and sends it via visited IMS to home IMS and ISCF where it is processed (could be authorized by home UPSF (8), and also apply service logic with interaction with ICAF (9).

(10) After successful authorization ISCF initiates resource reservation in Home and/or Visited network using IMS mechanisms available there towards RACS (11) and after then setup also control and delivery channels to UE (12). Finally Home ISCF sends response to UE (13) and initiates content delivery through IMCF via appropriate IMDFs (14) to UE (15-16).

Accessing Services. The SDSF in Visited Network should collect information from Visited Network about services 


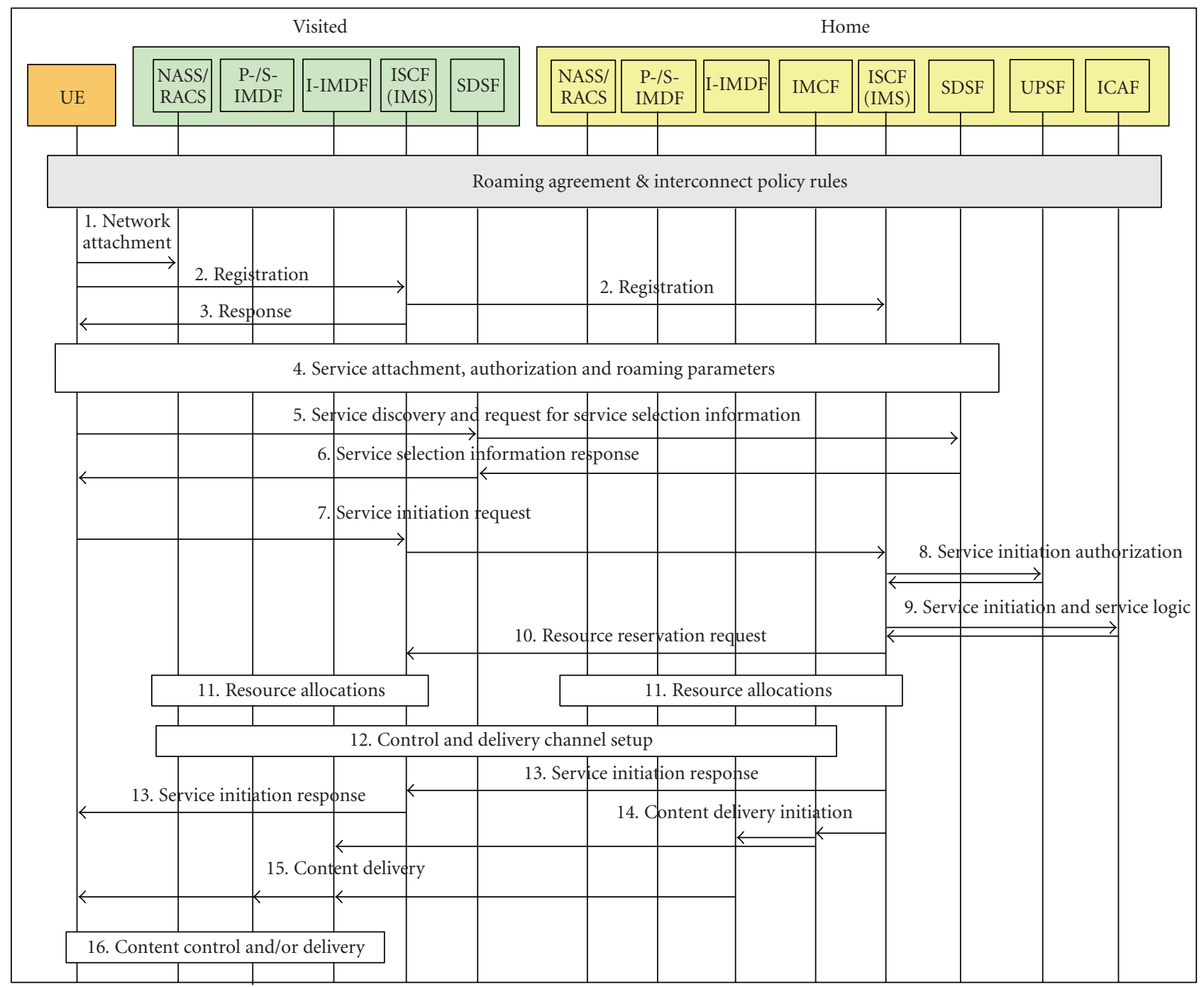

FIgURE 7: Procedures for interconnection with the Visited Network with CN-IPTV.

which subscriber can use in roaming but it also has access to relevant service information from Home Network. If this service is offered in Visited Network, the subscriber will get it directly from the Media distribution and Storage Subsystem of the Visited Network. If such service is not present in Visited Network, the UE will use it from the Media distribution and Storage Subsystem of Home network.

Since the UE or Visited Network can have some specific restrictions to format of content, the IMDFs in Visited Network must be able to transcode any stream to requested format and serve it to the UE. If there is no conjunction in functions of the Visited Network, I-IMDF, and UE possibilities, this media cannot be delivered to the customer. Therefore it is very useful for the IPTV providers in roaming relation to offer all the content in several bandwidth profiles (Mobile/WEB, SD, HD) with standardized common used codecs (3GPP, FLV, H.264, VC-1, MPEG2) preferably the same (to avoid transcoding). All UEs should support agreed and standardized codecs used in the Home Networks or during roaming in Visited Network.
TABLE 1: Examples of codec profiles for IPTV.

\begin{tabular}{lcccc}
\hline Profile & Video codec & Audio codec & Container & Bandwidth \\
\hline Mobile & H.263 & AAC & 3GPP & $256 \mathrm{kbit} / \mathrm{s}$ \\
WEB & H.264, baseline & mp4a & mp4 & $768 \mathrm{kbit} / \mathrm{s}$ \\
SD & H.264, main & MP2/AAC & MPEG2 TS & $3.5 \mathrm{Mbit} / \mathrm{s}$ \\
HD & H.264, high & MP2/DD+ & MPEG2 TS & $12 \mathrm{Mbit} / \mathrm{s}$ \\
\hline
\end{tabular}

Table 1 is just example, but worldwide discussion and agreement should be found and some global table should be proposed for interconnection agreements. This will help all service providers to save costs for technology and will increase interconnection possibilities.

To save the resources in the provider's networks, it is useful to define a closed group of bandwidths and codes used for the streaming, for example, as follows [12].

Content Elements from Many Sources. If there is a service in the Visited Network which is not the same as that one, 
which was requested from the UE, it is possible to combine content/service that partially get this content/service from local source and from Home Network source. If the video stream with some source (e.g., CNN TV) is present in the Visited Network, but language is different as requested one, the MDF can request only this elementary language. This process requires global synchronization information, which has to be put in stream (e.g., RTP timestamp).

4.2. Interconnect from Service Perspective. In the following section are discussed technical scenarios for selected IPTV services like Linear TV, Live TV with Trickplay, Timeshift $\mathrm{TV}$, and COD. We analyze scenarios and advantages from operator perspective and also user perspective especially when Visited network resources could be reused during roaming. Additionally two specific use cases for Advance PVR and UGC are explained as potentially attractive user services in roaming case. The main purpose for support of service roaming and terminal mobility is to enable to users the access to most of his personalized IPTV services and various contents from any terminal on the move with fixed terminal (e.g., nomadic mobility for moving STB from one location to other), portable devices (e.g., game console, laptops), or mobile (e.g., PDA, UMTS phone with DVB-H or MBMS/PSS).

4.2.1. Linear/Broadcast TV. Every channel of both operators must have a common channel identificator or at least synchronized metadata in both SDSFs. If the users can access some live channel which is present in visited network in the same quality, the SDSF must provide service selection information from the Visited Network. The provider of the Visited Network can agree with the Home Network provider that channels from the Visited Network can be offered to the subscriber (providing more channel); however, they are not a part of subscriber offer in the Home Network (e.g., some local channels provided for free or paid additionally to monthly fee). There should be other policies and addressing for multicast-based services in Home and Visited network or interconnect should not support multicast. In these cases IIMDF from home network can adapt service to unicast and I-IMDF in Visited transform address back to multicast or deliver media to UE by unicast.

4.2.2. Trickplay of Live TV Stored in Network. In the case of Live TV channel with trickplay it is reasonable to make use of the local resource from the Visited Network. Otherwise the operators must agree which network should be used to store the data needed for trickplay (Visited/Home). If the Visited Network has not enough space for trickplaying another channel or the operators of the Visited Network do not want to spend this space, it is necessary to offer the service from the Home Network. This solution spends the bandwidth on the interconnect interface. The P-IMDF in Visited Network asks the Home Network to deliver the stream for the trickplay recording and then the trickplay session will start to serve the unicast stream from Visited PIMDF to the UE.
4.2.3. Timeshift TV Service Served by Network. In the case of a channel which should be timeshifted (the channel with timeshift service also provided in the Visited Network), it is possible to use this resource from the Visited Network (caching/timeshifted in Visited PMDFs). Otherwise the operator of the Visited Network must store a huge amount of content from all the timeshifted channels from the Home Network operator offer, what can be unfeasible and wasting of resources and a subject for legislative problems. Therefore it is probable that this service will be offered from the Home Network. This will spend the bandwidth on the interconnect interface and can denied user request for timeshifting in roaming scenario.

4.2.4. CoD Service. Content on Demand (CoD) is very simple from the technical point of view, because it is defacto a catalogue and database of content (e.g., movie files/assets) and pure unicast delivery. If the UE searches CoD catalogue (e.g., in SDSF) and chooses the content which is accessible from the Visited Network, the UE will get it from this resource and do not need to consume the interconnect bandwidth. Otherwise content will be provided from Home Network IMDFs. If there are many visiting UEs from the same network and there is some popular content in it, it is useful to cache it anyway in the Visited Network. If there is any need for some adaptation of the content, this must be done by the transcoding in IMDF in prior to sending media to the UE over Visited Network.

4.2.5. Advanced PVR. If the UE requests recording (PVRpersonal video recording) of the content which is already stored in the Visited Network storage, it should be served from this resource. If there is not such a content available, the UE will get the content from the Home Network.

The UE requests to record a channel, it should be done in the Home Network, because there is high probability of subscriber's presence in the Home Network, and therefore it is playback in this network.

In special case there are channels offered only in Visited Network, here the nPVR should be done in Visited Network and playback should be possible only in this network. In the case of client-based PVR (c-PVR) it is independent from roaming because every time it is stored in UE.

We also specified specific use case where the combination of both PVRs (c-PVR with combination of n-PVR) could be provided called Advanced or Hybrid PVR.

Multiple scenarios or situations when client PVR is disabled to record show that (e.g., end device is disconnected in time of scheduled recording or network parameters are not sufficient to stream or missing capacity of storage in local device) IPTV solution should record in network and later deliver to end device. In the case of different end device capabilities (resolution, encoding, etc.), the record by nPVR can be done in several formats but appropriate record will be distributed to end device storage to later preview.

4.2.6. User Generated Content. All user contents must be stored in the Home Network. If the user wants to 
upload or upstream some content, the UE will open the upload/upstream channel to his home storage in P-IMDF in the Home Network and stores it there. The playback of this content should be done from the Home Network, too. Handling of popular content should be done the same way as described in CoD. Advantage of the supporting UGC in roaming scenarios is specially when user would like share, for example, holiday videos or content from Visited Network as UGC for friends in Home Network (if interconnect allowed).

\section{Conclusion}

Available services, their quality, reliability, usability, and conformability are the main drivers from the user to select service of the content provider, of course apart from the price. Standardized IPTV solution could enable the IPTV providers operate IPTV services with better utilization and in cost effective manner.

Only standardized solutions could provide the interoperability among solutions, operators, and end devices. Next step could be the interconnection of operators, and IPTV service providers in order to provide user's mobility and roaming across multiple domains and access technologies.

The paper has presented the concept of Converged NGNbased IPTV which aggregates the content from multiple sources and provides it to user's end devices over various access technologies and terminals. Some scenarios explaining the mobility, interconnection, and subscriber roaming scenarios within Converged NGN-based IPTV networks are also presented in the paper. Stress has been given to IPTV services behavior in interconnect scenarios and implication in order to use them in an effective way in roaming.

The IPTV interconnection is one of the actual topics in ETSI TISPAN where the work on technical report which has to analyze possible mapping with other IPTV systems, interconnection issues and roaming scenarios, and hybrid concept has been just started.

\section{Acknowledgments}

The first author is a senior designer in Slovak Telekom where he has been actively participating on $\mathrm{R} \& \mathrm{D}$ project ScaleNet and ETSI TISPAN NGN Release 2/3 standardization with more than 80 agreed contributions included in several TISPAN IPTV-related specifications (e.g., IPTV-related work items WI0005, WI2048, WI2049, WI3127, WI3137, WI7029, WI1059, WI2070, 2074, WI2079, and WI3208). Some parts of this paper are based on contributions of an author within ETSI TISPAN. This paper also presents some of the results from participation in various research projects at Slovak University of Technology in Bratislava such as NGNlab project [13], European CELTIC EUREKA project Netlab [14], Slovak National research projects: AV project 4/0019/07: Converged technologies for next generation networks (NGNs), and Slovak National basic research projects VEGA 1/0720/09 and VEGA 1/4084/07.

\section{References}

[1] Recommendation ITU-T Y.1910 (09/2008), "IPTV functional architecture," ITU-T, 2008.

[2] ETSI, “TISPAN; service layer requirements to integrate NGN services and IPTV," TS 181016 V2.0.0 (2007-11), ETSI, Sophia Antipolis Cedex, France, 2007.

[3] Draft ETSI, "TISPAN; IPTV architecture; NGN integrated subsystem for IPTV functions," TS 182028 V3.2.6 (2009-06), ETSI, Sophia Antipolis Cedex, France, 2009.

[4] Draft ETSI, "TISPAN; IPTV architecture; IPTV functions supported by the IMS subsystem," RTS 182027 V3.2.6 (200906), ETSI, Sophia-Antipolis Cedex, France, 2009.

[5] ETSI, "TISPAN; dedicated IPTV subsystem stage 3 specification,” Technical Specification 183064 V2.1.1 (2008-10), ETSI, Sophia Antipolis Cedex, France, 2008.

[6] ETSI, “TISPAN; IMS based IPTV stage 3 specification,” TS 183 063 V2.4.2 (2009-06), ETSI, Sophia Antipolis Cedex, France, 2009.

[7] 18bTD221r2_WI02074_NGN_Integrated_IPTV_migration, TISPAN 18 bis contribution, NGN integrated IPTVarchitecture \& migration scenarios, ETSI, 2008.

[8] E. Mikoczy, D. Sivchenko, B. Xu, and J. I. Moreno, "IPTV systems, standards and architectures_-part II: IPTV services over IMS: architecture and standardization," IEEE Communications Magazine, vol. 46, no. 5, pp. 128-135, 2008.

[9] ETSI, "DVB; transport of MPEG 2 based DVB services over IP based networks," Technical Specification 102034 V1.2.1, ETSI, Sophia Antipolis Cedex, France, 2006.

[10] DVB Document A128, "DVB-IP Phase 1.3 in the context of ETSI TISPAN NGN, DVB,” September 2008.

[11] OMA draft OMA-AD-BCAST-V1_0-20081209-C, "BCAST Mobile Broadcast Services Architecture," Candidate Version 1.0-2008, Open Mobile Alliance, 2008.

[12] ETSI, "DVB; specification for the use of video and audio coding in DVB services delivered directly over IP protocols," Technical Specification 102005 V1.3.1, ETSI, Sophia Antipolis Cedex, France, 2007.

[13] NGNlab-NGN laboratory, Slovak University of Technology in Bratislava, http://www.ngnlab.eu/.

[14] "NetLab: Use Cases for Interconnected Testbeds and Living Labs," http://www.celtic-initiative.org//Projects/NETLAB/ default.asp. 

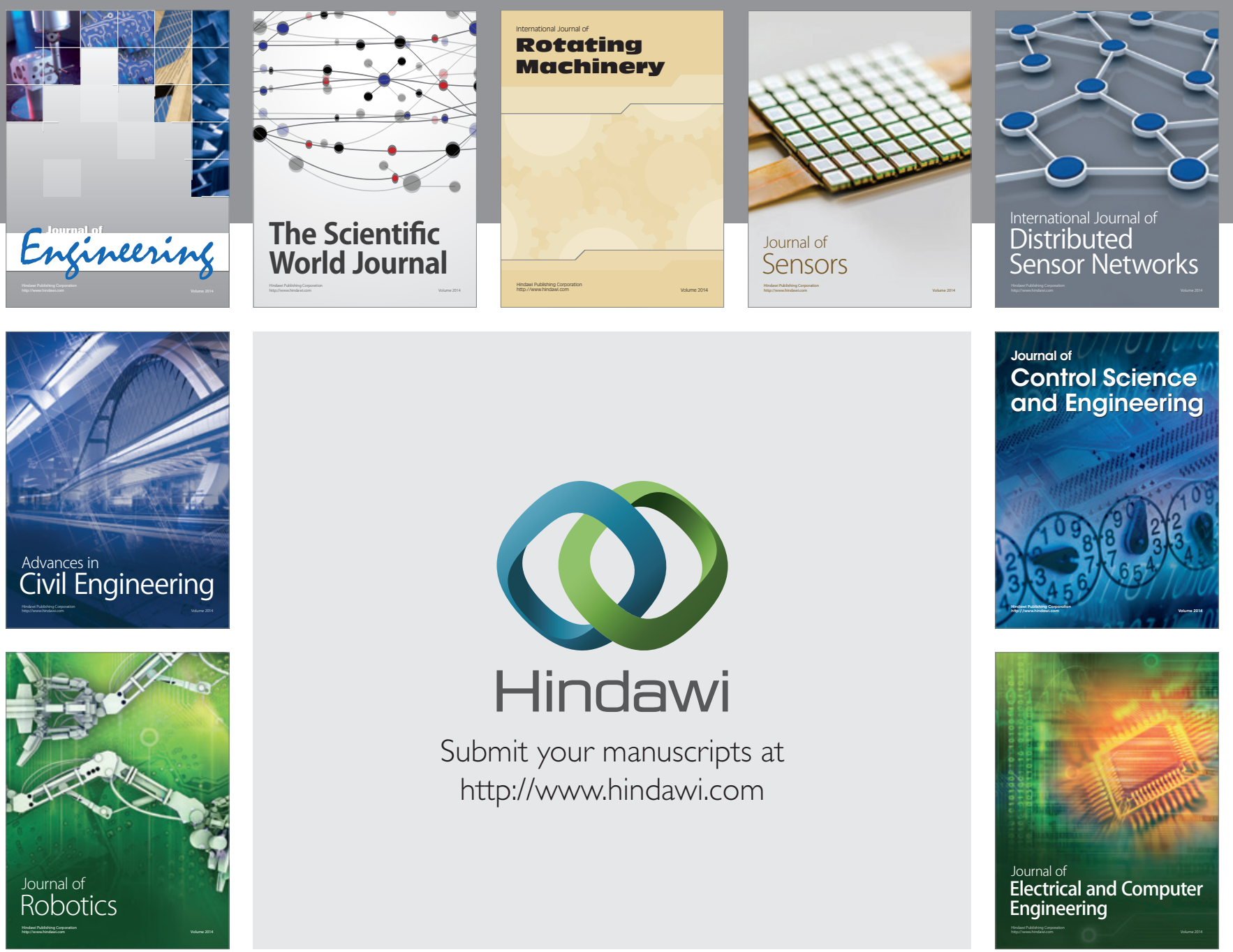

Submit your manuscripts at

http://www.hindawi.com
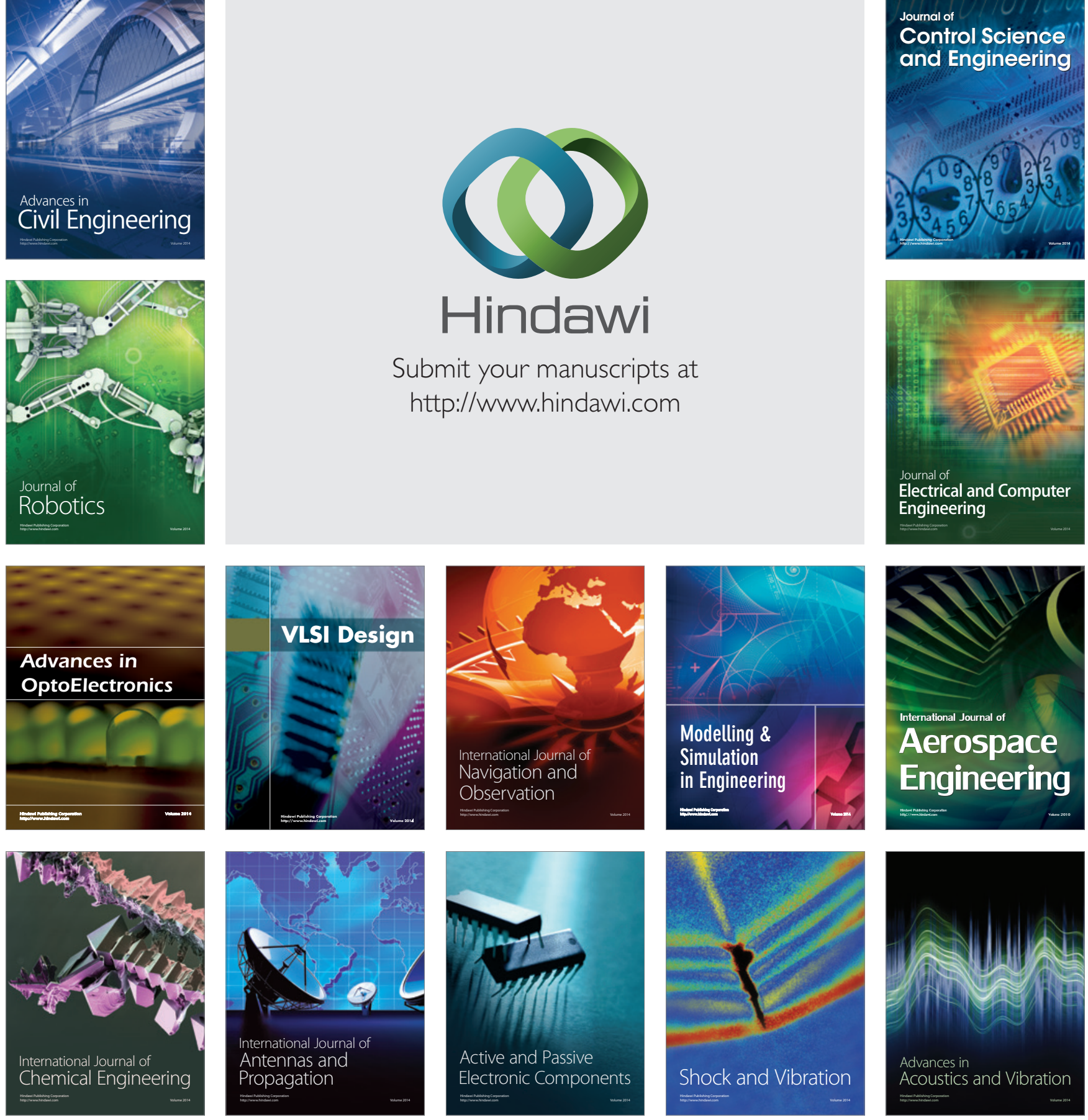